\title{
Epidemiology and species distribution of anaerobic Gram-negative cocci: a 10-year retrospective survey (2008-2017)
}

\author{
MÁRIÓ GAJDÁCS ${ }^{1,2 *}$, EDIT URBÁN ${ }^{3}$ \\ ${ }^{1}$ Department of Pharmacodynamics and Biopharmacy, Faculty of Pharmacy, University of Szeged, \\ 6720 Szeged, Eötvös utca 6., Hungary \\ ${ }^{2}$ Institute of Clinical Microbiology, Faculty of Medicine, University of Szeged, 6725 Szeged, Semmelweis utca 6., Hungary \\ ${ }^{3}$ Department of Public Health, Faculty of Medicine, University of Szeged, 6720 Szeged, Dóm tér 10., Hungary \\ *Corresponding author: Márió Gajdács \\ gajdacs.mario@pharm.u-szeged.hu
}

Received: 22 April 2019 / Revised: 5 June 2019 / Accepted: 12 June 2019 / Published online: 24 July 2019

\begin{abstract}
Introduction: The group of anaerobic Gram-negative cocci (AGNC) includes the genera Veillonella, Megasphaera, Anaeroglobus, Negativicoccus and Acidaminococcus. These bacteria are an integral part of the microbiome of humans but may be causative agents in various infectious processes. The available data on the epidemiology and significance of AGNCs is scarce.

Aims: To assess and compare the prevalence of different species of AGNCs among inpatients and outpatients at the Albert Szent-Györgyi Clinical Center retrospectively, during a 10-year study period.

Methods: Isolates containing AGNC were identified retrospectively by reviewing the online microbiology records of the Institute of Clinical Microbiology.

Results: The median age of affected patients overall was 52 years (range: 1-90 years), with a male dominance. 59.79\% of samples originated from inpatients. 572 individual AGNCs isolates were recovered from clinical samples, most of the isolated GNACs were Veillonella spp. (95.28\%), Megasphaera and Acidaminococcus species accounted for a minority of isolates (2.79\% and 1.93\%, respectively), while Anaeroglobus and Negativicoccus species were not isolated. In the second half of the study period (2013-2017), 91.31\% of isolates were identified on the species level ( $p<0.001)$ using MALDI-TOF MS.

Conclusion: The current study represents a long-term surveillance study on the isolation frequency and trends among anaerobic Gram-negative cocci (AGNCs), isolated in the Southern Great Plain of Hungary, highlighting the beneficial effect of MALDI-TOF MS on the diagnostic efficacy of the laboratories
\end{abstract}

Keywords: anaerobic bacteria, Veillonella spp., MALDI-TOF MS, clinical microbiology, epidemiology

\section{Introduction}

The group of anaerobic Gram-negative cocci (AGNC) includes the genera Veillonella, Megasphaera, Anaeroglobus, Negativicoccus and Acidaminococcus [1]. These bacteria (with Veillonella species in the highest numbers among the members of the group) are an integral part of the microbiome of the oral cavity and the gastrointestinal, genitourinary and respiratory tracts of humans [1,2]. Before the advent of molecular methods (polymerase chain reaction) and novel diagnostic techniques in the clinical microbiology laboratories (e.g., mass spectrometry, sequencing), identification of anaerobic Gram-negative cocci was based solely on Gram stain morphology and anaerobic cultivation [2-5]. Awareness regarding the roles of anaerobes in various infectious diseases has increased in recent years, especially due to the pronounced interest towards the study of the human microbiome; although the organisms mostly studied were
Clostridium, Bacteroides, Prevotella, Fusobacterium species and Gram-positive anaerobic cocci (GPAC) [6-8]. There is a distinct lack of data and an uncertainty regarding the prevalence and importance of AGNC species in human infections: these organisms are recovered in pure culture relatively infrequently, in addition, many laboratories do not have the suitable resources and equipment (e.g., GasPack jars, anaerobic glove boxes) for their isolation and identification [9,10]. The epidemiology of different pathogens shows great variation among time periods and geographic regions therefore, the assessment of local data is essential to evaluate trends over time and to reflect on the national situation, compared to international data. The aim of this study was to assess and compare the prevalence of different species of AGNCs among inpatients and outpatients at the Albert Szent-Györgyi Clinical Center (Szeged, Hungary) retrospectively, during a 10-year study period. 


\section{Materials and methods}

\subsection{Study design}

The Albert Szent-Györgyi Clinical Center is an 1,820-bed (1,465 active and 355 chronic beds, respectively), primary and tertiary care university-affiliated (University of Szeged) teaching hospital, servicing an urban and rural population in the southeast region of Hungary of about 405,000 people.

Isolates containing AGNC were identified retrospectively by reviewing the online microbiology records of the Institute of Clinical Microbiology. The data screening included samples taken at inpatient departments and outpatient clinics over a 10-year period (January 2008-December 2017). In addition, patient data was also collected, limited to demographic characteristics (age, sex, inpatient/outpatient status) and sample type. Isolates were considered separate if they occurred more than 30 days apart or different AGNCs were isolated [11,12].

\subsection{Identification of isolates}

The processing of samples arriving to the Institute of Clinical Microbiology was carried out according to guidelines in routine clinical bacteriology. The cultivation of anaerobic bacteria was carried out in line with principles in anaerobic bacteriology, at $37^{\circ} \mathrm{C}$ in an anaerobic chamber (Baker Ruskinn, York, UK). Between 2008 and 2012, the identification of anaerobic isolates was carried out based the presumptive methods recommended by the Wadsworth Anaerobic Bacteriology Manual, additionally, with the Rapid ID 32A (bioMérieux, Marcyl'Étoile, France) identification kit [13]. From 2013 onwards, identification was performed using matrixassisted laser desorption/ionization time-of-flight mass spectrometry (MALDI-TOF MS). Bacterial cells were transferred to a stainless-steel target using a sterile toothpick. An on-target extraction was performed by adding $1 \mu \mathrm{l}$ of $70 \%$ formic acid prior to the matrix. After drying at ambient temperature, the cells were covered with $1 \mu \mathrm{l}$ matrix HCCA $(\alpha-$ cyano-4-hydroxycinnamic acid in 50\% acetonitrile $/ 2.5 \%$ trifluoro-acetic acid). Mass spectrometry measurements were performed by the Microflex MALDI Biotyper (Bruker Daltonics, Bremen, Germany) in positive linear mode across the $\mathrm{m} / \mathrm{z}$ range of 2 to $20 \mathrm{kDa}$; for each spectrum, 240 laser shots at $60 \mathrm{~Hz}$ in groups of 40 shots per sampling area were collected [5]. The MALDI Biotyper RTC 3.1 software (Bruker Daltonics, Bremen, Germany) and the MALDI Biotyper Library 3.1 were used for spectrum analysis. Antibiotic susceptibility testing is not routinely performed from AGNC isolates $[14,15]$.

\subsection{Statistical analyses}

Descriptive statistical analysis (including means or medians with ranges and percentages to characterize data) was performed using Microsoft Excel 2013. Additional statistical analyses were performed with SPSS software version 24 (IBM SPSS Statistics for Windows 24.0, Armonk, NY, IBM Corp.), using the $\chi^{2}$-test and Mann-Whitney's U-test to compare groups. $\mathrm{P}$ values below 0.05 were considered statistically significant.

\section{Results and discussion}

\subsection{Study population}

The median age of affected patients overall was 52 years (range: 1-90 years), with a male dominance (56.64\%, with male-to-female ratio of 1.31 ), which was present is most of the individual study years (Table I). $59.79 \%$ of samples originated from inpa-

Table I General characteristics of the study population (grouped by study year)

\begin{tabular}{|c|c|c|c|c|c|}
\hline Study year & 2008 & 2009 & 2010 & 2011 & 2012 \\
\hline No. of affected patients & 19 & 29 & 27 & 21 & 39 \\
\hline Male-to-female ratio & 2.17 & 1.23 & 0.80 & 1.33 & 0.74 \\
\hline Percentage of inpatients & $57.89 \%$ & $68.97 \%$ & $48.15 \%$ & $61.90 \%$ & $58.97 \%$ \\
\hline Median age [yrs] & 55 & 50 & 47 & 56 & 52 \\
\hline Age range [yrs] & $(1-75)$ & $(6-87)$ & $(3-79)$ & $(18-90)$ & $(2-84)$ \\
\hline Study year & 2013 & 2014 & 2015 & 2016 & 2017 \\
\hline No. of affected patients & 30 & 44 & 68 & 134 & 159 \\
\hline Male-to-female ratio & 1.00 & 2.33 & 1.34 & 0.56 & 1.26 \\
\hline Percentage of inpatients & $63.33 \%$ & $65.00 \%$ & $69.12 \%$ & $63.43 \%$ & $52.20 \%$ \\
\hline Median age [yrs] & 54 & 55 & 55 & 49 & 51 \\
\hline Age range [yrs] & $(11-83)$ & $(4-84)$ & $(1-86)$ & $(8-81)$ & $(7-83)$ \\
\hline
\end{tabular}


Table II Origin of samples of interest at the Albert SzentGyörgyi Clinical Center (SZTE)

\begin{tabular}{c|c}
\hline Department/Clinic & $\%$ \\
\hline ENT & 29.29 \\
\hline Surgery & 16.07 \\
\hline Internal Medicine & 11.61 \\
\hline Oral Surgery & 10.89 \\
\hline Neurology & 0.71 \\
\hline Obstetrics and Gynecology & 3.21 \\
\hline Pediatrics & 3.93 \\
\hline Dermatology & 8.93 \\
\hline Intensive care & 5.71 \\
\hline Emergency & 1.61 \\
\hline Ophtalmology & 0.36 \\
\hline Traumatology & 5.71 \\
\hline Urology & 1.96 \\
\hline
\end{tabular}

ENT: Dept. of Otorhinolaryngology

tients. No statistically significant difference was observed between the patient characteristics (gender, age, inpatient/outpatient status; $\mathrm{p}>0.05$ ), however, a slight dominance of patients $\geq 50$ years of age could be noted in both the inpatient and outpatient groups $(58.48 \%$ for inpatients and $63.63 \%$ for outpatients, respectively).

Almost one-third of the samples originated from the Department of Otorhinolaryngology (29.29\%); other major contributors to positive samples include the Departments of Surgery (16.07\%), Internal Medicine 11.61\%) and Oral Surgery $(10.89 \%)$, while the remaining $32.14 \%$ of samples originated from nine other institutions (Table II).

$31.45 \%$ of isolated GNACs originated from abscess samples, while various wound isolates obtained by invasive sampling methods $(19.82 \%)$ or superficial swabbing (15.64\%) were also prevalent. Other positive samples include bile specimens, puncture samples from the sinuses and inner ear, bronchoalveolar lavage, blood cultures and intrauterine devices (IUD) (33.09\%). The composition of sample types that were positive for AGNCs is not surprising as most of these originated from anatomical regions, where these bacteria as present as normal flora, in addition to being relevant pathogens, if there is an underlying pathology or surgical intervention in the patient's anamnestic data.

During the 10-year study period, 572 individual AGNCs isolates were recovered from clinical samples at the Albert Szent-Györgyi Clinical Center. Most of the isolated GNACs were Veillonella spp. (95.28\%), including V. parvula and V. atypica (corresponding to more than half of the isolated species) as the most frequently isolated genera during the study period; in contrast, Megasphaera and Acidami- nococcus species accounted for only a minority of isolates $(2.79 \%$ and $1.93 \%$, respectively), while $A n$ aeroglobus and Negativicoccus species were not isolated (Table III). AGNCs were isolated as standalone pathogens in only $32.87 \%$ of cases, they were mostly isolated together with other bacteria, as part of a complex aerobic/anaerobic flora. The number of isolated AGNCs was significantly higher in the period after 2013 (136 vs. 436 isolates; $\mathrm{p}<0.001$ ). In the first half of the study period (2008-2012), most of the isolated pathogens were identified only at a genus level $(78.83 \%)$, while in the second half of the study period (2013-2017), 91.31\% of isolates were identified on the species level $(\mathrm{p}<0.001)$.

\section{Conclusions}

The current study represents a long-term surveillance study on the isolation frequency and trends among anaerobic Gram-negative cocci (AGNCs), isolated in the Southern Great Plain of Hungary over a 10-year period. To the best of our knowledge, this is the first and longest-spanning study reporting on their prevalence (and infections caused by these pathogens by proxy) in Hungary.

Based on the results of this retrospective survey, the most prevalent isolates at our tertiary-care center were Veillonella spp. (which may be considered the anaerobic counterparts of Neisseria species), while other species in the group only represented a fraction of the isolated species; this is in line with the findings of the few studies available in the literature. Hospitalization, a slight male dominance and the age ( $>50$ years) of many affected patients also correspond to literature findings $[1-3,5,10]$. There was a significant increase in both the number of isolated AGNC species and their correct (species-level) identification in the second half of the study period. Both may be explained by the introduction of the MALDI-TOF MS identification system to the diagnostic workflow of the laborato-

Table III Species distribution among GNACs in the study period (2008-2017)

\begin{tabular}{c|rc}
\hline Veillonella spp. & $\mathbf{5 4 5}$ & $\mathbf{( 9 5 . 2 8 \% )}$ \\
\hline V. parvula & 238 & $(41.60 \%)$ \\
\hline V. atypica & 103 & $(18.01 \%)$ \\
\hline V. dispar & 56 & $(9.79 \%)$ \\
\hline V. denticariosi & 5 & $(0.87 \%)$ \\
\hline V. ratti & 2 & $(0.35 \%)$ \\
\hline Megasphaera spp. & $\mathbf{1 6}$ & $\mathbf{( 2 . 7 9 \% )}$ \\
\hline Megasphera muciniformis & 10 & $(1.75 \%)$ \\
\hline Acidaminococcus spp. & $\mathbf{1 1}$ & $\mathbf{( 1 . 9 3 \% )}$ \\
\hline Acidaminococcus intestinum & 8 & $(1.39 \%)$ \\
\hline
\end{tabular}


ry. Several studies have reported on the beneficial changed MALDI-TOF put forth in clinical microbiology, both in regard to the speed and the quality of identification [16]. This is especially true with anaerobic bacteria, as their cultivation and identification with conventional methods is time- and resource-consuming $[5,9]$.

If we analyse these results from a clinical perspective, this would mean that 1-2 positive samples were received by the Institute of Clinical Microbiology per week, where significant growth of AGNCs was detected. In these cases, the continuous communication between physicians and the diagnostic microbiology laboratory is crucial. The role of the laboratory is to supply clinically relevant information in a precise and timely manner, which should be reciprocated by the feedback of the physicians, beginning from the submission of the sample, followed by information regarding the symptoms of the patient and the clinical picture [9].

Some limitations of this study must be acknowledged. Firstly, the presence and nature of symptoms of the patients are unknown; additionally, due to the inability to access the medical records of the individual patients affected, the correlation between the existence of relevant underlying illnesses (e.g., Type 2 diabetes, surgical interventions, iatrogenic or disease-related immunosuppression) and the infection could not be assessed. Furthermore, antimicrobial susceptibility testing of the isolated AGNC species was not performed, therefore no information is presented regarding the resistance trends in the isolated bacterial strains.

\section{Acknowledgements}

The authors would like to thank the lab technicians of the Institute of Clinical Microbiology for the excellent laboratory assistance. M.G. was supported by the National Youth Excellence Scholarship [Grant Number NTP-NTFÖ-18-C-0225] and the ESCMID Mentorship and Observership Programme.

\section{Competing interests}

The authors declare no conflict of interest, monetary or otherwise.

\section{References}

1. Tille P. Bailey and Scott's Diagnostic Microbiology. 14th ed. St. Louis: Mosby; 2018.
2. Brazier JS. Veillonella and Other Anaerobic GramNegative Cocci. In: Brian WJ Mahy (ed.): Topley \& Wilson's Microbiology and Microbial Infections. 1st ed. New Jersey: John Wiley \& Sons; 2010. https://doi. org/10.1002/9780470688618.taw0050

3. Finegold SM. Anaerobic Bacteria in Human Disease. New York: Academic Press; 1977.

4. La Scola B, Fournier PE, Raoult D. Burden of emerging anaerobes in the MALDI-TOF and 16S rRNA gene sequencing era. Anaerobe 2011; 17: 106-112. https://doi. org/10.1016/j.anaerobe.2011.05.010

5. Nagy E, Becker S, Kostrzewa M, Barta N, Urbán E. The value of MALDI-TOF MS for the identification of clinically relevant anaerobic bacteria in routine laboratories. J. Med. Microbiol. 2012; 61: 1393-400. https://doi. org/10.1099/jmm.0.043927-0

6. Siezen RJ, Kleerebezem M. The human gut microbiome: Are we our enterotypes? Microb. Biotechnol 2011; 4: 550-553. https://doi.org/10.1111/j.17517915.2011.00290.x

7. Bultman SJ. Emerging roles of the microbiome in cancer. Carcinogenesis 2014; 35: 249-255. https://doi. org/10.1093/carcin/bgt392

8. Finegold SM. State of the art; microbiology in health and disease. Intestinal bacterial flora in autism. Anaerobe 2011; 17: 367-368. https://doi.org/10.1016/j.anaerobe.2011.03.007

9. Gajdács M, Spengler, G, Urbán, E. Identification and Antimicrobial Susceptibility Testing of Anaerobic Bacteria: Rubik's Cube of Clinical Microbiology? Antibiotics 2017; 6: 25. https://doi.org/10.3390/antibiotics6040025

10. Nagy E. Anaerobic Infections Update on Treatment Considerations. Drugs 2010; 70: 841-858. https://doi. org/10.2165/11534490-000000000-00000

11. Urbán E. Five-year retrospective epidemiological survey of anaerobic bacteraemia in a university hospital and rewiew of the literature. Eur. J. Microbiol. Immunol. 2012; 2: 140-147. https://doi.org/10.1556/EuJMI.2.2012.2.7

12. Körmöndi S, Terhes G, Pál Z, Varga E, Harmati M, Buzás K, Urbán E. Human Pasteurellosis Health Risk for Elderly Persons Living with Companion Animals. Emerg. Infect. Dis. 2019; 25 (2): 229-235. https://doi. org/10.3201/eid2502.180641

13. Jousimies-Somer H, Summanen P, Citron DM, Baron EJ, Wexler HM, Finegold SM (ed.) Wadsworth-KTL Anaerobic Bacteriology Manual, 6th ed. Belmont, CA: Star Publishing Company; 2003.

14. Gajdács M. The Concept of an Ideal Antibiotic: Implications for Drug Design. Molecules 2019; 24: 892. https:// doi.org/10.3390/molecules24050892

15. Gajdács M, Paulik E, Szabó A. [The opinions of community pharmacists related to antibiotic use and resistance]. Acta Pharm. Hung. 88, 249-252.

16. Veloo AC, de Vries ED, Jean-Pierre H, Justesen US, Morris T, Urban E, Wybo I, van Winkelhoff AJ, ENRIA workgroup. The optimization and validation of the Biotyper MALDI-TOF MS database for the identification of Gram-positive anaerobic cocci. Clin. Microbiol. Infect. 2016; 22(9): 793-798. https://doi.org/10.1016/j. cmi.2016.06.016 